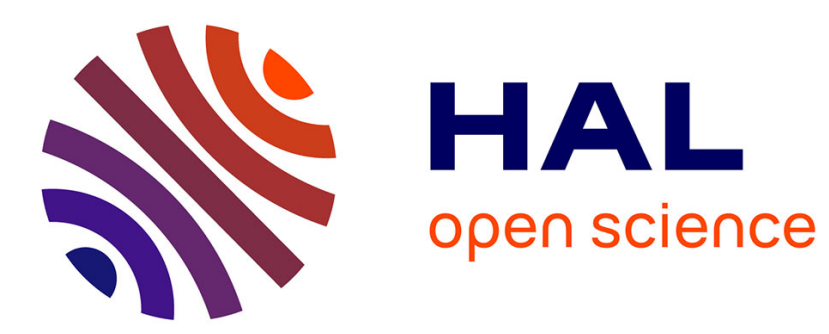

\title{
Téléphone et coordination des soins intensifs à l'hôpital
}

Carla Vaucher, Esther González-Martínez

\section{To cite this version:}

Carla Vaucher, Esther González-Martínez. Téléphone et coordination des soins intensifs à l'hôpital. La revue de l'infirmière, 2015, 64 (208), pp.38 - 39. 10.1016/j.revinf.2014.11.014 hal-01859226

\section{HAL Id: hal-01859226 https://hal.science/hal-01859226}

Submitted on 10 Sep 2018

HAL is a multi-disciplinary open access archive for the deposit and dissemination of scientific research documents, whether they are published or not. The documents may come from teaching and research institutions in France or abroad, or from public or private research centers.
L'archive ouverte pluridisciplinaire HAL, est destinée au dépôt et à la diffusion de documents scientifiques de niveau recherche, publiés ou non, émanant des établissements d'enseignement et de recherche français ou étrangers, des laboratoires publics ou privés. 


\section{Téléphone et coordination des soins intensifs à l'hôpital}

\author{
Carla Vaucher \\ Assistante de recherche \\ Dr Esther \\ González-Martínez* \\ Professeur HES \\ Haute école de santé Arc, \\ Haute école spécialisée \\ de Suisse occidentale, \\ Espace de l'Europe 11, \\ CH-2000 Neuchâtel, Suisse
}

Le téléphone est un outil central pour la coordination des soins à l'hôpital entre les services de soins généraux et les soins intensifs. Les appels sont brefs, fréquents. Ils concernent une grande diversité de questions traitées en parallèle à d'autres activités. Cette recherche, réalisée par une équipe suisse, examine les pratiques de communication téléphonique en milieu hospitalier.

(c) 2014 Elsevier Masson SAS. Tous droits réservés

Mots clés - communication ; coordination ; infirmière ; soins intensifs ; Suisse ; téléphone

Telephone and coordination of intensive care in hospital. The telephone is a central tool for the coordination of care in hospitals between the general wards and intensive care. Calls are short and frequent. They concern a wide variety of issues handled in parallel with other activities. This research, carried out by a Swiss team, examines the practices of telephone communication in hospitals.

(c) 2014 Elsevier Masson SAS. All rights reserved

Keywords- communication; coordination; intensive care; nurse; Switzerland; telephone

a coordination téléphonique des soins à l'hôpital a fait l'objet d'une étude suisse [1]. Ont été recueillis des appels entre le service de chirurgie et d'autres services d'un même hôpital de Suisse romande, et réalisées des observations ainsi que des entretiens.

Les soins intensifs sont un interlocuteur important des infirmières de chirurgie. Les appels concernent des questions variées, exigent précision et rapidité, et sont souvent réalisés en parallèle à d'autres activités. Nos observations contribuent à la réflexion sur l'organisation des soins et les compétences communicationnelles nécessaires au travail infirmier en milieu hospitalier [2].

\section{Une organisation complexe}

\section{Plusieurs téléphones}

$\checkmark$ Le bureau des infirmières

*Auteur correspondant. Adresse e-mail : esther.gonzalezmartinez @he-arc.ch

(E. González-Martínez) de chirurgie de l'hôpital étudié dispose d'un téléphone fixe. Celles-ci utilisent également un téléphone portable, confié à l'infirmière de référence pour la journée, distinct du téléphone portable de l'infirmière chef.

४ Le personnel des soins intensifs utilise, quant à lui, deux téléphones fixes pour les communications avec les autres services, un téléphone portable pour la communication interne au service, ainsi qu'un téléphone fixe exclusivement réservé aux situations de réanimation. Les infirmières chef et les médecins des deux services disposent également de téléphones personnels fixes et/ou portables.

- La diversité d'appareils et leur utilisation incessante témoignent de l'importance de la communication téléphonique ainsi que de ses différentes fonctions.

\section{Une activité parmi \\ d'autres}

\ Le téléphone est utilisé de manière complémentaire et souvent simultanément à d'autres moyens de communication. Par exemple, il arrive régulièrement que les infirmières s'appellent pendant qu'elles consultent ensemble des informations médicales sur un ordinateur. II arrive également qu'elles avancent au téléphone des informations qui seront complétées en face-à-face ultérieurement, lors des transmissions.

$\downarrow$ En outre, la communication téléphonique vient souvent se greffer à d'autres activités en cours, réalisées par les membres des équipes soignantes, tenus de répondre à tout moment. Les appareils fixes sont d'ailleurs stratégiquement placés de manière à être accessibles rapidement, par exemple des deux côtés du desk des soins intensifs, mais également à proximité d'ordinateurs, moniteurs et dossiers utilisés fréquemment.

\ La communication téléphonique mobilise ainsi des compétences certaines de gestion de l'information et de multi-activité, d'autant plus qu'elle se fait dans des espaces communs, où les interruptions sont fréquentes et où règne un bruit de fond permanent 
(conversations, sonnettes, alarmes). De plus, les appels sont souvent réalisés par le personnel en situation de déplacement.

\section{Caractéristiques et contenu des appels Rapides, synthétiques et cordiaux}

Les appels entre les infirmières de chirurgie et les soins intensifs durent en moyenne 50 secondes, une durée très similaire à celle des appels avec d'autres services. Ils sont donc très brefs et synthétiques, souvent concentrés sur une affaire à la fois. Malgré leur brièveté, les conversations comportent toujours une ouverture et une clôture, lors desquelles les interlocuteurs s'identifient et échangent des salutations.

- Au travers de formats subtilement modulés, de "voix souriantes" ou de rires ténus, les interlocuteurs introduisent de la cordialité dans les appels sans pour autant les prolonger. Soigner la relation interpersonnelle entre collègues semble particulièrement important lors d'appels où des demandes sont à satisfaire rapidement, alors que toutes sortes de contingences sont fréquentes.

\section{En rapport avec les soins}

$\checkmark$ Les communications téléphoniques sont initiées plus fréquemment par les soins intensifs que par les infirmières de chirurgie, soucieuses de ne pas interrompre l'activité d'un service traitant de situations d'urgence vitale.

$\checkmark$ Les appels sont de nature professionnelle et concernent principalement le transfert des patients, mais aussi des demandes de conseils au sujet de l'administration d'un médicament ou l'accomplissement d'un soin, le prêt de matériel ou encore l'envoi d'objets ou de documents.

\section{Le transfert}

des patients

४ L'arrivée ou le départ d'un patient des soins intensifs implique souvent plusieurs conversations téléphoniques. Par exemple, les soins intensifs appellent pour fixer l'heure exacte à laquelle l'infirmière de chirurgie doit aller chercher un patient et lui indiquent déjà par téléphone les examens à effectuer avant de le conduire dans le service. Ceci permet à l'infirmière d'estimer au plus près le temps nécessaire au transfert et le moment du retour du patient. Lorsque l'équipe des soins intensifs est prête pour le transfert, elle rappelle la chirurgie.

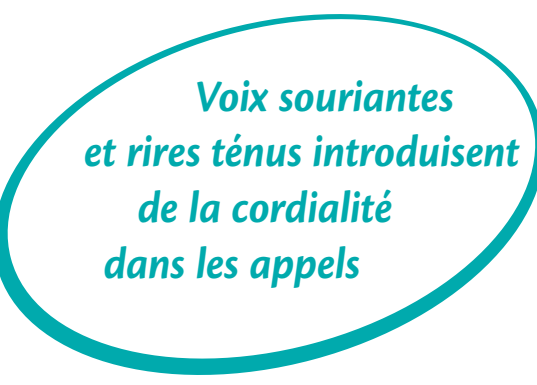

- Parfois, l'infirmière appelle pour s'assurer de la disponibilité de l'équipe ou de la possibilité d'avancer ou de retarder quelque peu le transfert. Elle peut également s'enquérir des objets et documents à prendre avec elle.

- Le transfert entraîne également des communications nécessaires au suivi des soins, ainsi que la transmission complète du dossier du patient et de ses affaires personnelles.

\section{Le souci du temps}

- Les communications téléphoniques visent la prise en charge optimale du patient ainsi que la gestion des ressources en termes de personnel et de lits disponibles. Les interlocuteurs posent ainsi des délais très précis lors des transferts des patients et procèdent à des rappels lorsque

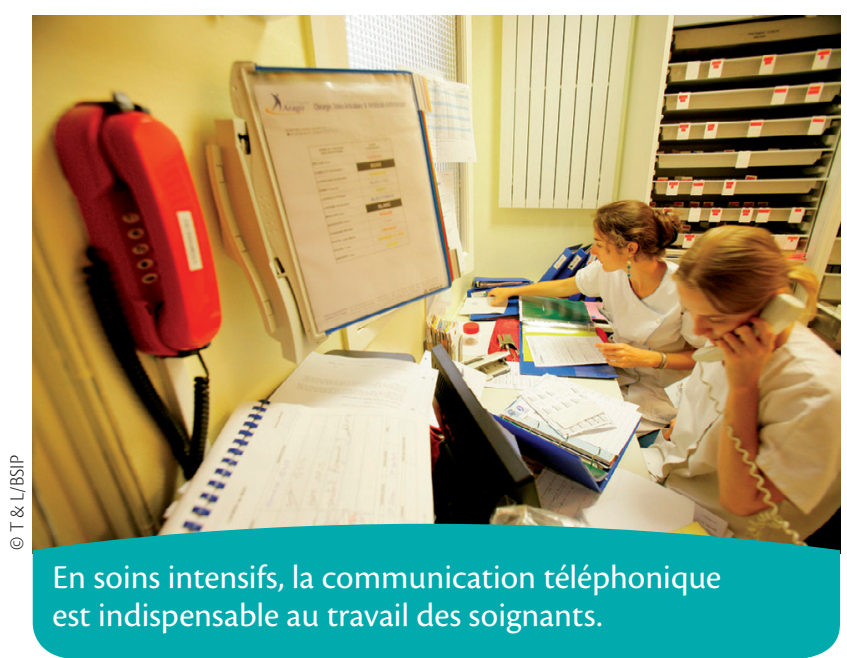

ceux-ci ne sont pas strictement respectés ou qu'il est nécessaire de les modifier, même de manière minime

$\downarrow$ La gestion du temps se révèle être un souci constant pour les soignants, en ce qui concerne la durée des appels et leur contenu.

\section{Conclusion}

Le développement de nouvelles technologies de communication hospitalière rend indispensable la production de connaissances quant à leur utilisation et à leur articulation, fondées sur l'observation de situations de travail concrètes [3].

Pour l'infirmière aguerrie, la coordination téléphonique des soins peut paraître de l'ordre de l'évidence. Toutefois, pour l'infirmière débutante, il s'agit d'une activité qui n'est ni à redouter ni à banaliser, à laquelle il est particulièrement difficile de s'exercer dans les institutions de formation.

Elle exige des compétences spécifiques : être synthétique et rapide tout en communicant de manière compréhensible, fiable et cordiale. Ces aptitudes sont particulièrement importantes dans le cas des communications avec les soins intensifs gérant des situations d'urgence vitale. •

\section{Références}

[1] Nouvelle dans le poste: pratiques de mise en pertinence et d'appréciation des compétences professionnelles des jeunes nfirmières lors de leurs conversations téléphoniques à I'hôpital. Projet Sinergia IC-You, subside du Fonds national suisse de la recherche scientifique $\mathrm{n}^{\circ}$ 136291/1, Université de Neuchâtel www2.unine.ch/ic-you/page22675.html

[2] Gajo L. Langue de I'hôpital, pratiques communicatives et pratiques de soins. Lausanne: Cahiers de l'Institut de linguistique tes sciences du langage 2004 16: $1-6$

[3] Coiera E, Tombs V.

Communication behaviours in a hospital setting. An observational study. BMJ 1998; 316 (7132): 673-6.

Remerciements

Nous remercions le personnel de l'Hôpital Neuchâtelois de sa participation à notre recherche.

Déclaration d'intérêts Les auteurs déclarent ne pas avoir de conflits d'intérêts en relation avec cet article. 